\title{
Revisiting lactic acidosis in an HIV-1 infected pregnant woman on antiretroviral therapy
}

N. Mendes ${ }^{1,2}$, L. Aboím¹ , A. Borges 3 , C. Guerreiro ${ }^{1} \&$ C. Castelo-Branco ${ }^{4}$

${ }^{1}$ Department of Obstetrics and Maternal-Fetal Medicine, Centro Hospitalar de Lisboa Central, Maternidade Dr. Alfredo da Costa, ${ }^{2}$ Nova Medical School, Universidade Nova de Lisboa, Lisbon, Portugal ${ }^{3}$ Department of Internal Medicine, Centro Hospitalar de 
Lisboa Central, Maternidade Dr Alfredo da Costa, and ${ }^{4}$ Obstetrics, Gynaecology and Neonatology Clinic Institute, Hospital Clinic, University of Barcelona, IDIBAPS, Barcelona, Spain

\section{DOI: $10.3109 / 01443615.2012 .734344$}

Correspondence: N. Mendes, Department of Obstetrics and Maternal-Fetal Medicine, Centro Hospitalar de Lisboa Central, Maternidade Dr. Alfredo da Costa, Rua de Viriato, 1069-089 Lisbon, Portugal. E-mail: neuzamdm@ hotmail.com

\section{Introduction}

Mitochondrial toxicity induced by inhibition of gamma DNA polymerase is an adverse effect of nucleoside reverse transcriptase inhibitors (NRTI) and is responsible for a broad spectrum of clinical conditions that include lactic acidosis and hepatic steatosis (Brinkman et al. 1998). Growing data suggest increased susceptibility during pregnancy (Fleischer et al. 2004). Moreover, fatal cases have been reported during the 3rd trimester and with long-term therapy (Luzzati et al. 1999; Sarner and Fakoya 2002; Bristol-Myers Squibb 2001). Hence, the combination of stavudine and didanosine is currently not recommended throughout gestation (Bristol-Myers Squibb 2001; AIDS info 2012).

We report a case of an HIV-1-infected pregnant woman presenting in the 1st trimester, with symptoms suggesting lactic acidosis and who had been on stavudine and lamivudine for only 5 months.

\section{Case report}

A 27-year-old HIV-1-infected nulliparous woman presented with vomiting, nausea, asthenia and anorexia in the 10th week of pregnancy. She had been on antiretroviral (ARV) therapy since the age of 22. At 3 months before pregnancy, and because her viral load was rising, she was switched to stavudine, lamivudine and lopinavir/ ritonavir. At the time of conception, she had a CD4 count of $641 \times 10^{6} / 1$ and an undetectable viral load $(<50$ copies $/ \mathrm{ml})$. On admission, examination revealed apyrexia, and a normal pulse and respiratory rate. There were no focal signs of hepatitis, pancreatitis or obstetric complications. Investigations showed an AST 21 times the upper limit of the norm (ULN); ALT 13.5 times the ULN; an elevated GGT and LDH (7.5 and $5 \times$ ULN, respectively) and a metabolic acidosis $(\mathrm{pH}=7.32)$, with low bicarbonate $\left(\mathrm{HCO}_{3}\right)$ and elevated arterial lactate $(2.5 \times \mathrm{ULN})$ (Table I). Abdominal ultrasound and other tests were in the normal range. She was managed with the diagnosis of lactic acidosis and liver dysfunction probably secondary to NRTI.

ARV therapy was immediately stopped. She was managed with close surveillance, monitoring gasometry and lactate levels, daily. The patient was symptom free within 3 days and discharged after 15 days in hospital, during which time investigations gradually returned to normal (Table I). Viral load increased to 175.190 copies/ $\mathrm{ml}$ and CD4 dropped to $221 \times 10^{6} / \mathrm{l}$.

ARV therapy was restarted 4 weeks later, using zidovudine, lamivudine and lopinavir/ritonavir. Subsequently, AST and ALT remained within the normal range throughout the rest of the pregnancy and viral load decreased, becoming undetectable before delivery. An elective caesarean section was performed at 38 weeks and 3 days. The apparently healthy infant was also given zidovudine, according

Table I. Analytical developments throughout admission.

\begin{tabular}{lrrrrrrrr}
\hline & D1 & D2 & D5 & D7 & D9 & D11 & D13 & D15 \\
\hline AST (IU/l) & 631 & 621 & 441 & 271 & 197 & 112 & 72 & 32 \\
ALT (IU/l) & 402 & 383 & 329 & 256 & 182 & 126 & 88 & 28 \\
GGT (IU/l) & 307 & 321 & 354 & 582 & 450 & 316 & 216 & 89 \\
LDH (IU/l) & 2412 & 2405 & 1828 & 1435 & 1408 & 1242 & 1084 & 595 \\
pH & 7.32 & 7.38 & 7.36 & 7.33 & 7.34 & 7.32 & 7.38 & 7.38 \\
HCO $_{3}(\mathrm{mmol} / \mathrm{l})$ & 12.6 & 12.6 & 17.8 & 17.5 & 17.6 & 16.9 & 20 & 17.9 \\
Lactate (mg/dl) $^{4}$ & 47 & 38 & 40 & 45 & 47 & 43 & 33 & 31 \\
\hline
\end{tabular}

D, day of hospitalisation; AST, aspartate aminotransferase; ALT, alanine aminotransferase; GGT, gamma glutamyl transferase; $\mathrm{LDH}$, lactate dehydrogenase, $\mathrm{HCO}_{3}$, bicarbonate. to hospital protocol. DNA PCR HIV tests were negative at 48 hours and 2, 4, 6 and 12 months after birth.

\section{Discussion}

The incidence of NRTI-induced lactic acidosis/hepatic steatosis in HIV-infected pregnant women is unknown. Nevertheless, there are three reported cases of maternal death related to stavudine and didanosine use, two associated with fetal death (Bristol-Myers Squibb 2001; Sarner and Fakoya 2002). Non-fatal cases with this combination have also been described (Mandelbrot et al. 2003). There is also a reported case of fetal death at 38 weeks' gestation, with stavudine and lamivudine (Luzzati et al. 1999). In all cases, the women presented after at least 6 months of therapy, with a rapidly progressive disease occurring in the $3 \mathrm{rd}$ trimester. This is in contrast to our case, in which the onset of the symptomatic disease was in the 1 st trimester and only after 5 months of stavudine and lamivudine.

NRTI-induced lactic acidosis/hepatic steatosis have similarities to rare but life-threatening syndromes: acute fatty liver of pregnancy (AFLP) and the HELLP syndrome (haemolysis, elevated liver enzymes and low platelets). Data support that both of these are associated with a recessively inherited condition that compromises fatty acid oxidation. The same dysfunctional pathway may contribute to the development of NRTI-induced mitochondrial toxicity (AIDS info 2012). In our case report, early gestational age and the absence of specific clinical and laboratory features excluded HELLP and AFLP. Therefore, the diagnosis of NRTI-induced lactic acidosis seemed most likely.

Low levels of riboflavin may also be involved in the pathogenesis of NRTI-induced mitochondrial toxicity (Luzzati et al. 1999). Lower levels of this vitamin have been reported in pregnant women compared with non-pregnant women (Vir et al. 1981). Moreover, riboflavin has been used to treat lactic acidosis (Mandelbrot et al. 2003; Brinkman et al. 2000). Likewise, other vitamin B complex elements have been used as therapeutic agents (thiamine, nicotinamide, pyridoxine and dexpanthenol), as well as co-enzyme Q, 1carnitine and vitamin C (Brinkman et al. 2000). Nevertheless, data in this regard is scarce to support its efficacy. Indeed, the treatment of this syndrome remains obscure and incidental. Most authors agree on the immediate interruption of antiretroviral drugs and the use of nonspecific adequate support measures.

As the benefit of ARV prophylaxis in reducing transmission of a fatal infection has long been clear, and the combination of stavudine and didanosine always seems to play a central role in the more serious reported cases of lactic acidosis, we decided to restart ARV therapy, replacing stavudine by zidovudine. The described derangement did not recur, probably because, as happens in vitro and also in vivo, the relative potency of stavudine in inhibiting mitochondrial gamma DNA polymerase is higher than that of zidovudine.

As pregnancy may enhance HIV-infected women's susceptibility to nucleoside analogues lactic acidosis, healthcare professionals should be vigilant for its onset. Nonspecific symptoms can easily be undervalued, especially in the 1 st trimester. However, they may represent the initial manifestation of a life-threatening syndrome and hence, always require further investigation.

\section{Acknowledgements}

We thank Professor Dr Kamal Mansinho of the Department of Infectious Diseases of Egas Moniz Hospital, Lisbon, Portugal.

Declaration of interest: The authors report no conflicts of interest. The authors alone are responsible for the content and writing of the paper.

\section{References}

AIDS info. 2012. Recommendations for the use of antiretroviral drugs in pregnant HIV-1-infected women for maternal health and interventions to reduce perinatal HIV transmission in the United States. Available at: http://aidsinfo. nih.gov/guidelines (Accessed 26 April 2012).

Brinkman K, Hofstede HJM, Burger DM et al. 1998. Adverse effects of reverse transcriptase inhibitors: mitochondrial toxicity as a common pathway. AIDS 12:1735-1744. 


\section{Obstetric Case Reports}

Brinkman K, Vrouenraets S, Kauffman R et al. 2000. Treatment of nucleoside reverse transcriptase inhibitor-induced lactic acidosis. AIDS 14:2801-2802.

Bristol-Myers Squibb Company. 2001. Healthcare provides important drug warning letter (5 January).

Fleischer R, Boxwell D, Sherman KE. 2004. Nucleoside analogues and mitochondrial toxicity. Clinical Infectious Diseases 38:e79-e80.

Luzzati R, Del Bravo P, Di Perri G et al. 1999. Riboflavin and severe lactic acidosis. Lancet 353:901-902.

Mandelbrot L, Kermarrec N, Marcollet A et al. 2003. Case report: nucleoside analogue-induced lactic acidosis in the third trimester of pregnancy. AIDS 17:272-273.

Sarner L, Fakoya A. 2002. Acute onset lactic acidosis and pancreatitis in the third trimester of pregnancy in HIV-1 positive women taking antiretroviral medication. Sexually Transmitted Infections 78:58-59.

Vir S, Love A, Thompson W. 1981. Riboflavin status during pregnancy. American Journal of Clinical Nutrition 34:2699-2705. 